\title{
Compact wide stopband microstrip lowpass filter using polygon patches and meandered lines
}

\author{
Adel Musavy, Akram Sheikhi \\ Department of Electrical Engineering, Lorestan University, Iran
}

\begin{tabular}{|c|c|}
\hline Article Info & ABSTRACT \\
\hline Article history: & In this paper, a low pass filter based on T-Shaped resonator is presented. The \\
\hline & T-Shaped resonator consists of meandered lines and rectangular patches. \\
\hline Received Dec 12, 2019 & Also, the LC model and transfer function of the proposed resonator is \\
\hline Revised Jan 2, 2020 & presented. For suppression of spurious harmonics, a bandstop structure \\
\hline Accepted Jan 21, 2020 & consists of hexangular patches and open stubs has been utilized. Finally, the \\
\hline Keywords: & $\begin{array}{l}\text { been simulated, fabricated and measured. The LPF has good characteristics } \\
\text { such as wide stopband and insertion loss lower than } 0.18 \mathrm{~dB} \text { in the passband }\end{array}$ \\
\hline Lowpass filter & region. The rejection level is less than $-20 \mathrm{~dB}$ from 2.98 up to $21.3 \mathrm{GHz}$. The \\
\hline Meandered line & filter size is $10.5 \mathrm{~mm} \times 12.7 \mathrm{~mm}$, or $0.131 \lambda_{\mathrm{g}} \times 0.158 \lambda_{\mathrm{g}}$, where $\lambda_{\mathrm{g}}$ is the guided \\
\hline $\begin{array}{l}\text { Patches } \\
\text { Pats }\end{array}$ & $\begin{array}{l}\text { wavelength. The measured and simulated results of the filter is in good } \\
\text { agreement with each other, which show the merits of low insertion loss and }\end{array}$ \\
\hline T-shaped resonator & wide stopband. \\
\hline
\end{tabular}

This is an open access article under the CC BY-SA license.

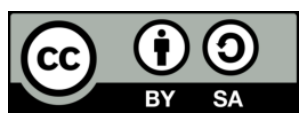

\section{Corresponding Author:}

Akram Sheikhi,

Department of Electrical Engineering,

Lorestan University,

P. O. Box 465, Khoramabad, 68137, Iran.

Email: akram.sheikhi2008@gmail.com

\section{INTRODUCTION}

The lowpass filters (LPFs) are used in the communication systems for elimination of undesired frequencies. There are several requirements for a LPF such as small size, low loss in passband region, and wide stopband. Planar filters have attracted considerable attention due to low cost, simple structures and capability of integration with other microwave circuits [1]. To suppress the spurious frequencies, the stepped impedance filters were introduced in [2-3]. To have high order harmonic suppression, stepped impedance hairpin resonator was proposed in [2]. In [3], a compact low pass filter with widestop band by using transformed stepped impedance resonator, was proposed. However, the reported work in [3] has poor selectivity from passband to stopband region. Other methods such as defected ground structure (DGS) and slotted structure to design of filters have been proposed, but the main problem is complexity of fabrication process [4-7]. In [4], the bandwidth of filter has been increased by a DGS structure. The filter based on DGS in [5] has wide stopband. Yet, it provides high insertion loss and gradual cutoff frequency. Also, multistopband filter based on tree fractal slotted structure in [6] and LPF using DGS in [7] have been proposed. The microstrip lowpass filters with compact size and ultrawide stopband using triangular and polygonal patch resonator are presented in [8] which have poor selectivity. In [9], multimode resonators to design of wide stopband LPF with high selectivity have been used. However, the multimode LPF has the stopband region with low attenuation level. In [10], a novel design of a LPF based on metamaterial was introduced. The proposed filter is investigated based on square split ring resonators (SRRs) with good attenuation level in the stopband but suffers from low stop bandwidth and large size. In [11], the presented filter used meandered 
lines, and polygonal patches with low passband insertion loss which suffer from low selectivity and relatively low rejection level in the stopband region.

To deal with problems such as low selectivity, narrow stopband, high insertion loss, and large size, a new microstrip lowpass filter with good performance has been presented in this work. This paper, addresses all those problems by obtaining wide stopband by bandstop structure instead of cascaded resonators since caseded resonators result in size increasing. From the proposed design, it is found that there is only optimum number of elements to maximize filter stopband and minimize passband losses.

The rejection level better than $-20 \mathrm{~dB}$ is obtained in the range of 2.98 up to $21.3 \mathrm{GHz}$. The filter size is $10.5 \mathrm{~mm} \times 12.7 \mathrm{~mm}$, or $0.131 \lambda_{\mathrm{g}} \times 0.158 \lambda_{\mathrm{g}}$, where $\lambda_{\mathrm{g}}$ is guided wavelength. Measured results of the developed lowpass filter are in good agreement with the simulation ones, thereby proving the validity of the proposed filter. The paper is organizaed as the following. In the first section, we provide an overview of the proposed resonator with lumped and distributed elements. In the second section, we argue the bandstop structure based on lumped and distributed elements. The LPF design and the obtained results are presented in third and fourth sections, respectivey. Finally, the conclusion is presented in the fifth section.

\section{DESIGN OF RESONATOR}

A new T-shaped resonator using meandered line is presented. Meandered lines are employed and loaded with rectangular patches to achieve the high selectivity and low insertion loss. In Figure 1(a) and (b), the layout and equivalent circuit of basic resonator consists of meandered line and rectangular patch is shown.

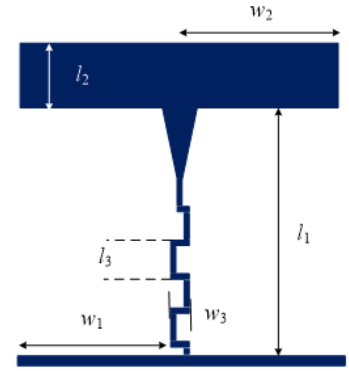

(a)

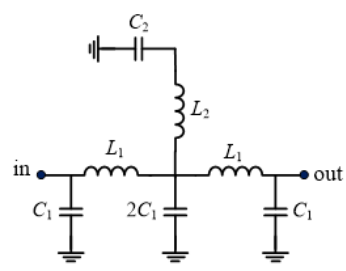

(b)

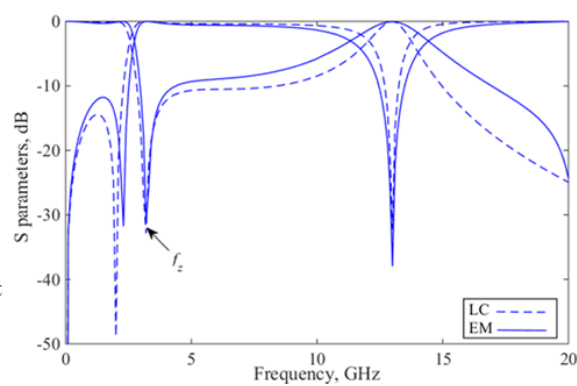

(c)

Figure 1. (a) Layout, (b) Equivalent circuit, and (c) Simulated $S$-parameters

In Figure 1(b), $L_{1}$ and $C_{1}$ are inductance and capacitance of high impedance transmission lines. $L_{2}$ is inductance of meandered line, which is assumed lossless, and $C_{2}$ refers to the capacitance of the rectangular patch. The values of inductors and capacitors can be obtained from (1)-(3) as

$$
L_{i}=\frac{1}{\omega} Z_{s i} \sin \left(\frac{2 \pi}{\lambda_{g}} l_{i}\right)
$$

and

$$
C_{i}=\frac{1}{\omega} \frac{1}{Z_{s i}} \tan \left(\frac{\pi}{\lambda_{g}} l_{i}\right)
$$

and the capacitance of the open stub is

$$
C_{o i}=\frac{\sqrt{\varepsilon_{e f f}}}{c Z_{s i}} l_{i}
$$

where $Z_{\mathrm{si}}$ is the characteristic impedance of the line, $l_{i}$ is its length, $c$ is the velocity of light in free space and $\lambda_{\mathrm{g}}$ is the guided wavelength at the cut-off frequency. The values of these parameters after optimization obtained as $L_{1}=3.1 \mathrm{nH}, L_{2}=3.05 \mathrm{nH}, C_{1}=0.07 \mathrm{pF}$, and $C_{2}=0.8 \mathrm{pF}$. The dimensions are $l_{1}=5.25 \mathrm{~mm}, l_{2}=1 \mathrm{~mm}$, $l_{3}=0.6 \mathrm{~mm}, w_{1}=3.9 \mathrm{~mm}, w_{2}=4.1 \mathrm{~mm}$, and $w_{3}=0.4 \mathrm{~mm}$. Figure $1(\mathrm{c})$ shows the LC and EM simulation results of the basic resonator. As it is illustrated, the agreement between LC and EM simulation results has been met perfectly. The $S_{21}$ parameter of the basic resonator shows that the resonator has low rejection level in the stopband. Also, the transmission zero (TZ) of the basic resonator is $3.15 \mathrm{GHz}$, which is obtained as 
$T_{1}(s)=\frac{Z_{0}\left(C_{2} L_{2} s^{2}+1\right)}{C_{1} C_{2} L_{1} L_{2} s^{4}+\left(L_{1}\left(C_{1}+C_{2}\right)+C_{2} L_{2}\left(2-Z_{0}\right)\right) s^{2}+\left(2-Z_{0}\right)}$

Hence, the position of transmission zero, $f_{\mathrm{z}}$, can be determined by setting the $T_{1}(\mathrm{~s})=0$, which results in $f_{\mathrm{z}}=3.3 \mathrm{GHz}$. Also, the variation of cut-off frequency of the basic resonator versus $w_{2}$ and $l_{2}$ parameters is shown in Figure 2(a) and 2(b), respectively. As it can be seen, the cutoff frequency has been decreased by increasing $w_{2}$ and $l_{2}$. By varying the dimensions of the patch structure, the cutoff frequency of the proposed resonator can be tuned.

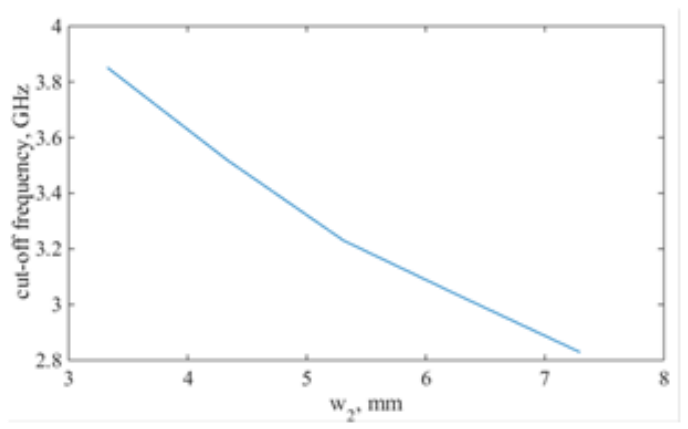

(a)

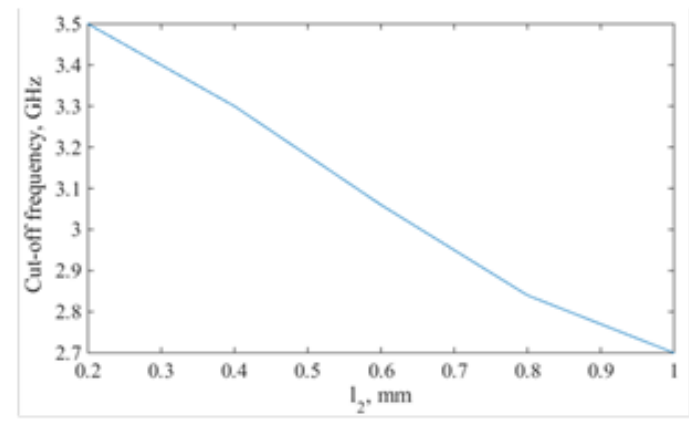

(b)

Figure 2. Cut-off frequency versus (a) $w_{2}$ and (b) $l_{2}$

Figure 3(a) and 3(b) show the proposed resonator and its equivalent LC circuit. The S-parameters of the proposed resonator and LC model is shown in Figure 3(c). It can be seen from Figure 3(d), the transmission zero is shifted from $4.6 \mathrm{GHz}$ up to $3.3 \mathrm{GHz}$ by increasing $w_{2}$. The transfer function (TF) of the proposed resonator is obtained as

$$
T_{2}(s)=\frac{Z_{0}\left(C_{2} L_{2} s^{2}+1\right)}{C_{1} C_{2} L_{1} L_{2} s^{4}+\left(L_{1}\left(C_{1}+2 C_{2}\right)+C_{2} L_{2}\left(2-Z_{0}\right)\right) s^{2}+\left(2-Z_{0}\right)}
$$

From $(5), f_{\mathrm{z}}=3.3 \mathrm{GHz}$ is obtained.

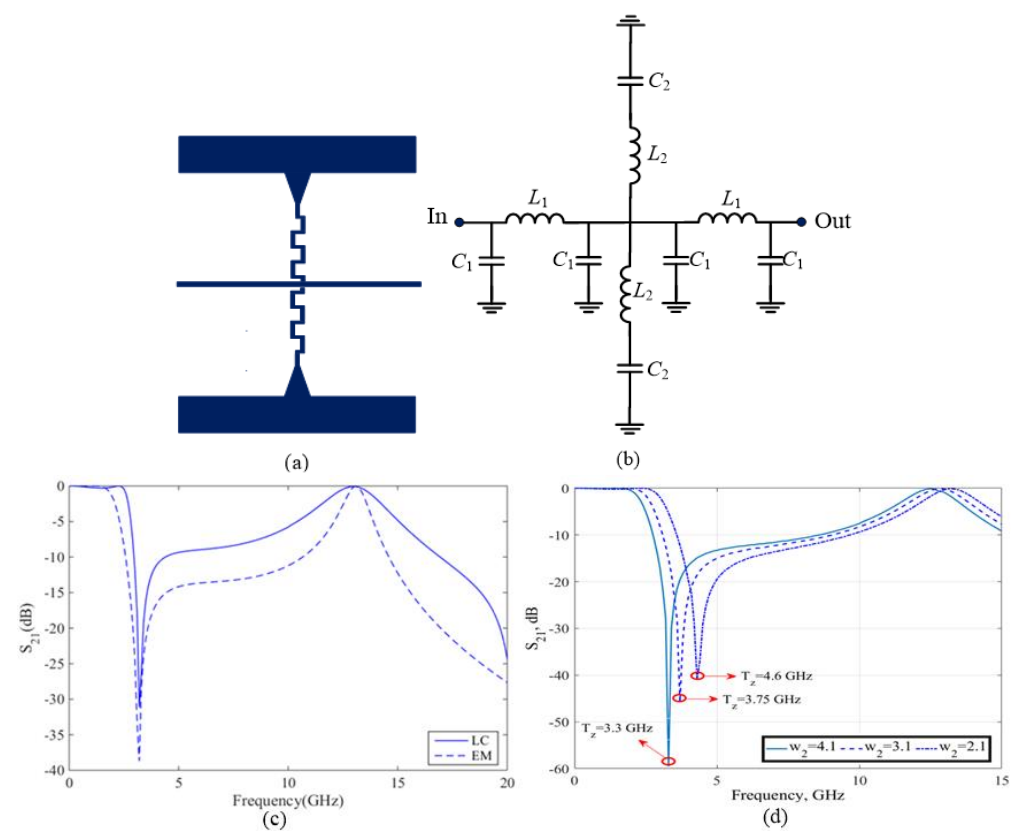

Figure 3. (a) Layout, (b) LC equivalent circuit, (c) Simulated S-parameters, and (d) simulated $\mathrm{S}_{21}$ parameter versus $w_{2}$ 


\section{DESIGN OF BANDSTOP STRUCTURE}

The bandstop structure (BSF) are designed based on two open stubs and high impedance line loaded by four hexangular patches (resonator-II). The main section is a high impedance line having an impedance of $134 \Omega$ connected in series with a hexangular patch. The resonator-II has been designed and simulated with the Advanced Design System (ADS). When the layout of the resonator has been generated, an electromagnetic momentum (EM) simulation has been carried out to analyze its performance. Its layout and LC equivalent circuit is shown in Figure 4. The dimensions are $l_{4}=3.13 \mathrm{~mm}$, and $l_{5}=1.7 \mathrm{~mm}$. This unit has one transmission zero at $8 \mathrm{GHz}$ with attenuation level $-43 \mathrm{~dB}$.

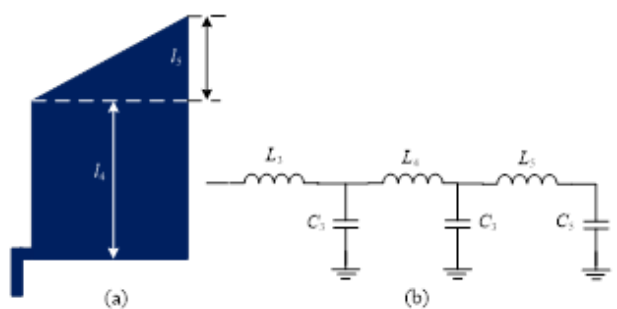

Figure 4. (a) Layout, and (b) Equivalent circuit of resonator-II

The configuration, LC equivalent circuit and simulated characteristics of two units are shown in Figure 5(a), 5(b) and 5(c), respectively. For the analysis of equivalent circuit, the hexangular patch has equivalent circuit of $C_{3}, L_{4}, L_{5}$, and $C_{5}$. The high impedance line is represented as $L_{3} . C_{3}$ and $C_{5}$ represent the capacitance between microstrip hexangular patch and the ground plane. The optimized values of LC equivalent circuit are $L_{3}=0.052 \mathrm{nH}, L_{4}=0.31 \mathrm{nH}, L_{5}=0.18 \mathrm{nH}, C_{3}=0.5 \mathrm{pF}$, and $C_{5}=0.37 \mathrm{pF}$. The transfer function of the two units of resonator-II is obtained as the following:

$$
T_{3}(s)=\frac{Z_{1} Z_{11}}{Z_{0}\left(Z_{1}+Z_{2}+Z_{11}\right)+Z_{11}\left(Z_{1}+Z_{2}\right)}
$$

where

$$
\begin{aligned}
& Z_{1}(s)=\frac{C_{3}^{2} C_{5} L_{3} L_{4} L_{5} s^{6}+\left(C_{3}^{2} L_{3} L_{4}+C_{3} C_{5} L_{3}\left(L_{4}+2 L_{5}\right)+C_{3} C_{5} L_{4} L_{5}\right) s^{4}+\left(C_{3}\left(2 L_{3}+L_{4}\right)+C_{5}\left(L_{3}+L_{4}+L_{5}\right)\right) s^{2}+1}{s\left(2 C_{3}+C_{5}+\left(C_{3}^{2} L_{4}+C_{3} C_{5} L_{4}+2 C_{3} C_{5} L_{5}\right) s^{2}+C_{3}^{2} C_{5} L_{4} L_{5} s^{6}\right)} \\
& Z_{11}(s) \\
& =\frac{2 C_{3}^{2} C_{5} L_{3} L_{4} L_{5} s^{6}+\left(2 C_{3}^{2} L_{3} L_{4}+2 C_{3} C_{5} L_{3}\left(L_{4}+4 L_{5}\right)+C_{3} C_{5} L_{4} L_{5}\right) s^{4}+\left(C_{3}\left(4 L_{3}+L_{4}\right)+C_{5}\left(2 L_{3}+L_{4}+L_{5}\right)\right) s^{2}+1}{s\left(2 C_{3}+C_{5}+\left(C_{3}^{2} L_{4}+C_{3} C_{5} L_{4}+2 C_{3} C_{5} L_{5}\right) s^{2}+C_{3}^{2} C_{5} L_{4} L_{5} s^{6}\right)}
\end{aligned}
$$

From (6), $f_{\mathrm{z} 1}=7.9 \mathrm{GHz}$ and $f_{\mathrm{z} 2}=8.5 \mathrm{GHz}$ is obtained. By connecting two units symmetrically connected at the centre of main high impedance transmission line, the structure in Figure 6 (a) is obtained. Figure 6(b) exhibits two transmission zeros, which are located at about $7.67 \mathrm{GHz}$ and $9 \mathrm{GHz}$ with attenuation level near $-62 \mathrm{~dB}$ and $-60 \mathrm{~dB}$. These transmission zeros are caused by the resonance of the high impedance single stepped stub loaded by triangular patches and its resonant frequency depends on the structural parameters.

In practical applications, a filter with wide stopband and high suppression level is necessary. The bandwidth of the proposed filter can be enhanced by connecting open stubs on both sides of the resonators. The layout and frequency response of two open stubs are shown in Figure 7. The open stub produces a transmission zero to eliminate the harmonic at $14 \mathrm{GHz}$. The filter using open circuit stub at both ends of the high impedance main transmission line exhibits better stopband characteristics. The length and width of the open stub are $l_{5}=10.2 \mathrm{~mm}$ and $w_{4}=1.17 \mathrm{~mm}$, respectively. Each open stub can be considered as a shunt capacitor to the ground. At high frequencies, the capacitive reactance decreases, and open stub acts as a short circuit to the ground. This shorts out transmission and causes attenuation in the stopband which suppresses the higher frequency harmonics and extends the stopband.

The bandstop unit in Figure 8(a) has been added to the resonator. The $S_{21}$ parameters of bandstop structure are depicted in Figure 8(b). At high frequency, the inductance of main high impedance line blocks transmission by having infinite series reactance, whereas the capacitance of open stub shorts out transmission by having infinite shunt susceptance. The rejection level from 4 up to $14 \mathrm{GHz}$ is better than $-15 \mathrm{~dB}$. So, the nondesired passband at $14 \mathrm{GHz}$ has been diminished. This clearly indicates the ability of the bandstop strcuture to elimination of unwanted harmonics and improved stop bandwidth. 


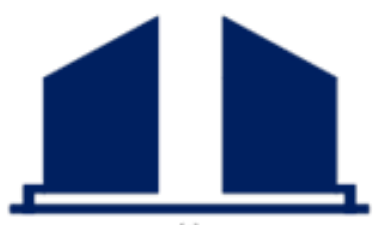

(a)

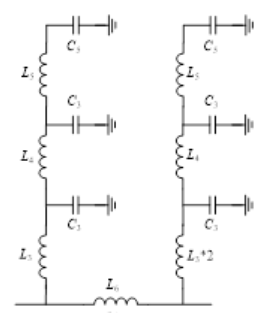

(b)

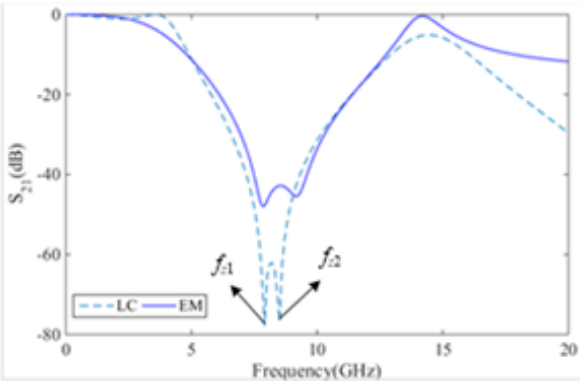

(c)

Figure 5. (a) Layout, (b) LC equivalent circuit, and (c) the simulation results of two units of resonator-II

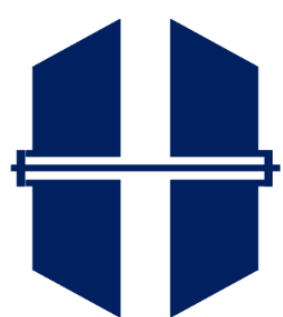

(a)

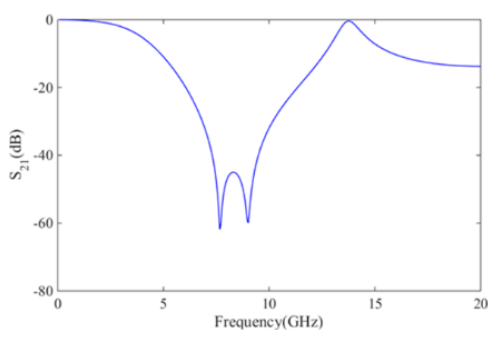

(b)

Figure 6. (a) Layout, and (b) the simulation results of two symmetrical structure of two units of resonator-II

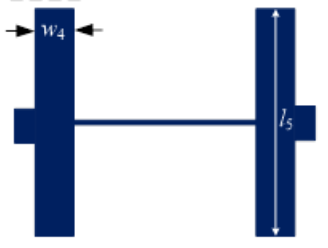

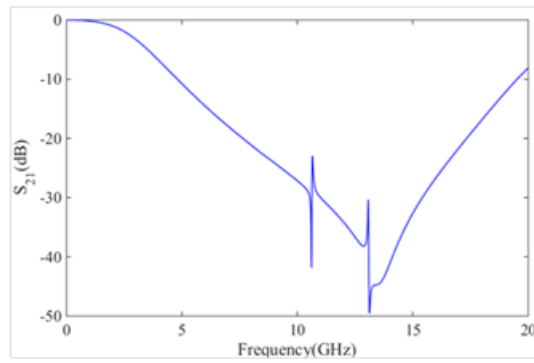

(b)

Figure 7. (a) Layout, and (b) Simulated $S_{21}$ parameter of two open stubs

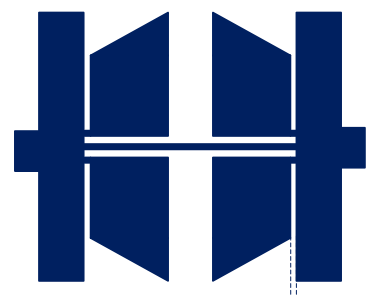

$\mathrm{g}=0.1 \mathrm{~mm}$

(a)

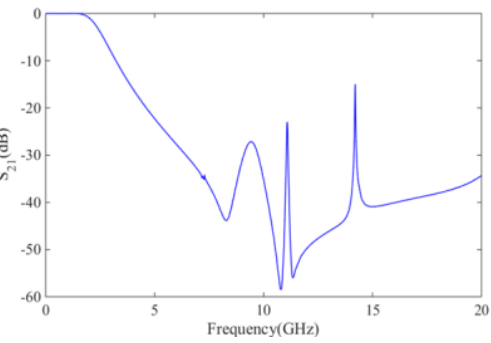

(b)

Figure 8. (a) Layout, and (b) Simulated $\mathrm{S}_{21}$ parameter

\section{FILTER DESIGN AND LAYOUT}

The schematic showing the generation process of the proposed filter on substrate with the permittivity of 2.2, loss tangent of 0.0009 and thickness of $0.508 \mathrm{~mm}$ is given in Figure 9(a). The LPF occupies $10.5 \mathrm{~mm} \times 12.7 \mathrm{~mm}$. Fig 9(b) shows the photograph of the fabricated filter. Addition of bandstop structure to the proposed resonator results in good rejection level in the stopband region. The proposed 
resonator was embedded between spaces of the bandstop structure to avoid size increasing of the filter. Conventional microstrip filters are usually implemented using cascaded resonator and bandstop structure which results in size increasing and losses. The filter was simulated by ADS 2014 and measured by HP8510B Network Analyzer. The measured and simulated results will be presented in the next section. Two microstrip lines connected at both sides of the LPF are in order to match the impedance at the input and output ports to $50 \Omega$ with the $W_{\mathrm{f}}=0.7 \mathrm{~mm}$, and $l_{\mathrm{f}}=2.4 \mathrm{~mm}$.

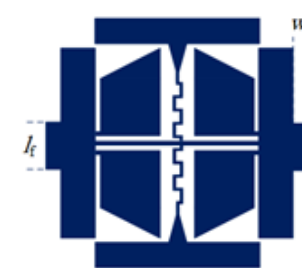

(a)

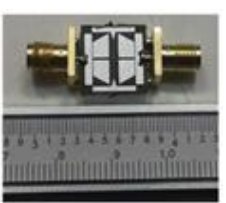

(b)

Figure 9. (a) Layout, and (b) test board

\section{RESULTS AND DISCUSSION}

Figure 10(b) shows the measured and simulated S-parameters of the proposed LPF. One transmission zero is located at $2.97 \mathrm{GHz}$ to have high selectivity. In addition, extra transmission zeroes in the stopband region can be observed. In general, these transmission zeros help us in out-of-band rejection enhancement. The measured insertion loss is less than $0.18 \mathrm{~dB}$, while the return loss is greater than $12.5 \mathrm{~dB}$ from DC up to $3.39 \mathrm{GHz}$. The simulated group delay is shown in Figure 10(c). It can be seen that the group delay is less than $0.32 \mathrm{~ns}$ in the passband. Furthermore, over $20 \mathrm{~dB}$ attenuation level in the stopband region is achieved from 2.98 up to $21.3 \mathrm{GHz}$, so that the unwanted signal is eliminated. For comparisons, Table 1 illustrates the measured results for some previuos works and proposed filter, where roll off rate $\zeta$ is defined as

$$
\zeta=\frac{\alpha_{\max }-\alpha_{\min }}{f_{c^{-}} f_{s}}
$$

where $\alpha_{\max }$ and $\alpha_{\min }$ represent the $-20 \mathrm{~dB}$ and $-3 \mathrm{~dB}$ attenuation level, respectively, $f_{\mathrm{c}}$ is the $-3 \mathrm{~dB}$ cutoff frequency, and $f_{\mathrm{s}}$ is the $-20 \mathrm{~dB}$ stopband frequency. In this case, the bandwidth divided by center frequency of stopband is the relative stopband width (RSB) as

$$
R S B=\frac{\text { stopband bandwidth }}{\text { stopband centre frequency }}
$$

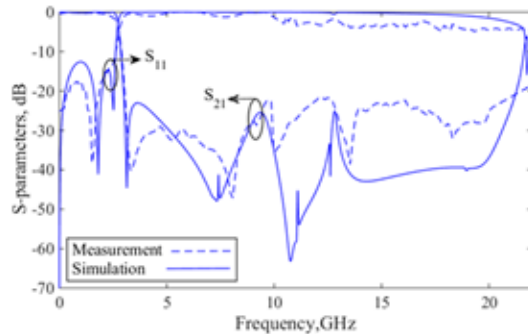

(a)

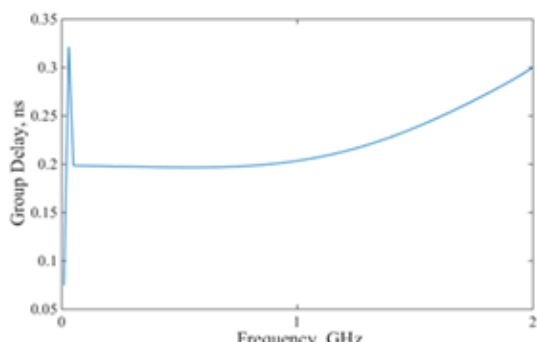

(b)

Figure 10. (a) Simulated and measured S-parameters, and (b) simulated group delay of the proposed filter

The rejection level divided by 10 is suppression factor (SF), which is defined as

$$
S F=\frac{\text { rejection level }}{10}
$$

As an example, the SF of the proposed LPF with rejection levels of $20 \mathrm{~dB}$ is 2 . The normalized circuit size (NCS) is defined as 
$N C S=\frac{\text { physical size (length } \times \text { width) }}{\lambda_{g}^{2}}$

Comparing with the other filters [2-3], [5], [7-11], clearly a transmission zero close to the passband is realized with T-shaped resonator and the filter has sharp roll-off rate in comparison to [2-3], [5], [7-11]. Moreover, the stop bandwidth of the proposed filter can be further extended by using Split Ring Resonators as [7]. Also, the proposed filter has lower insertion loss in comparison to other works. However, the filter is symmetrical and miniaturized in dimension which is less costly to implement in communication applications. The measured and simulated results of the filter have good agreement at lower frequency. The difference at high frequencies is due to the imperfect soldering of the ports and inaccurate implementation of the proposed filter.

Table 1. Comparison of the proposed LPF with other works.

\begin{tabular}{|c|c|c|c|c|c|}
\hline Ref & $\zeta$ & RSB & SF & Insertion Loss & NCS \\
\hline$] 2[$ & 52.8 & 1.53 & 2 & 0.3 & $0.113 \times 0.081$ \\
\hline$] 3[$ & 14.1 & 1.54 & 1.4 & 0.4 & $0.103 \times 0.071$ \\
\hline [5] & 43.9 & 1.355 & 1.5 & 1 & $0.15 \times 0.101$ \\
\hline$] 7[$ & 20 & 1.65 & 1.7 & 0.3 & $0.12 \times 0.071$ \\
\hline$] 8[$ & 16.66 & 1.55 & 1.5 & 0.36 & $0.081 \times 0.089$ \\
\hline$] 9[$ & 15 & 1.58 & 1.5 & 0.5 & $0.11 \times 0.09$ \\
\hline [10] & 17 & - & 2 & 0.2 & $0.11 \times 0.11$ \\
\hline [11] & 56.7 & 1.267 & 2 & 0.3 & $0.116 \times 0.115$ \\
\hline This work & 65.4 & 1.54 & 2 & 0.18 & $0.158 \times 0.131$ \\
\hline
\end{tabular}

\section{CONCLUSION}

An LPF with cutoff frequency $2.72 \mathrm{GHz}$ by modified T-shaped resonator has been designed, fabricated, and measured. To improve the stopband bandwidth, the bandstop structure consists of using open stubs and hexangular patches has been added at the two sides of the proposed resonator. An approximate LC equivalent circuit of each structure has been derived and its response has been compared with simulated result. A high attenuation over the stopband up to $21.3 \mathrm{GHz}$ has been obtained so that the unwanted out-ofband signal is eliminated. The obtained results show that the proposed filter has good performance such as low insertion loss, wide stopband and high selectivity in comparison to other filters. Due to compact size, low insertion loss and wide stopband, the proposed LPF is expected to be applied in modern wireless communication systems.

\section{REFERENCES}

[1] J. S. Hong and M. J. Lancaster, "Microstrip Filters for RF/Microwave Applications," New York, Wiley, 2001.

[2] S. Liu, J. Xu, and Z. Xu, "Compact lowpass filter with wide stopband using stepped impedance hairpin units," Electronic Letters., vol. 51, pp. 67-69, 2014.

[3] F. Zhang, S. Lio, P. Zhao, M. Du, X. Zhang, and J. Xu, "Compact ultra-wide stopband lowpass filter using transformed stepped impedance hairpin resonator," 2017 IEEE International Symposium on Antennas and Propagation \& USNC/URSI National Radio Science Meeting, 2017.

[4] S. Azizi, M. E. Halaoui, A. Kaabal, S. Ahyoud and A. Asselman, "Enhanced Bandwidth of Band Pass Filter Using a Defected Microstrip Structure for Wideband Applications," International Journal of Electrical and Computer Engineering (IJECE), vol. 8, no. 6, pp. 5260-5267, Dec. 2018.

[5] F. Wei, L. Chen, and X-W. Shi, "Compact lowpass filter based on coupled-line hairpin unit," Electronic Letters, vol: 48, pp. 379-381, 2012.

[6] S. K. Parui, S. Das, "Microstrip multi-stopband filter based on three fractal slotted resonator," International Journal of Electrical and Computer Engineering (IJECE), vol. 9, pp. 3657-3663, 2019.

[7] S. Sen, T. Moyra, and D. Sarkar, "Modelling and Validation of Microwave LPF Using Modified Rectangular Split Ring Resonators (SRR) and Defected Structure," International Journal of Electronics and Communication, Accepted for publication, 2018.

[8] H. Cui, J. Wang, and G. Zhang, "Design of microstrip lowpass filter with compact size and ultra-wide stopband," Electronics Lett, vol. 48, no. 14, pp 856-857, July 2012.

[9] Q. Li, Y. Zhang and Y. Fan., "Compact ultra-wide stopband low pass filter using multimode resonators," Electronic Letters, vol. 51, pp.1084-1085, 2015.

[10] B. Nasiri, A. Errkik, J. Zbitou, A. Tajmouati, L. Abdellaoui, and M. Latrach, "A Compact Planar Low-Pass Filter Based on SRR-Metamaterial," International Journal of Electrical and Computer Engineering (IJECE), vol. 86, pp. 4972-4980, 2018.

[11] L. Ge, J. P. Wang, and Y.-X. Guo, “Compact microstrip lowpass filter with ultra-wide stopband," Electronic Letters, vol. 46, 689-691, 2010. 